\title{
Can positron emission tomography- computed tomography-based three target lesions' total lesion glycolysis predict therapeutic response in Hodgkin Lymphoma?
}

\author{
Hend Yehia Ali ${ }^{* *}$, Shaimaa Abdelsattar Mohammad², Ali Hagag Ali², Ahmed Mohamed Monib² and \\ Mennatallah Hatem Shalaby ${ }^{2}$
}

\begin{abstract}
Background: Universally maximum standardized uptake value (SUVmax) and lactate dehydrogenase (LDH) are used as tools for response assessment in Hodgkin Lymphoma $(\mathrm{HL})$ patients. Our objectives are to evaluate the predictive potential and response assessment of total lesion glycolysis (TLG) and metabolic tumor volume (MTV) — maximum three target lesions - as another alternatives and to investigate the correlation between TLG and MTV with LDH.

Results: Both initial SUVmax and TLG were significantly associated with early patient response ( $p$ value $0.03,0.047$, respectively). An optimal threshold for SUVmax and TLG less than or equal 19.52, and 158.6, respectively, correlated with better therapeutic response. Initial LDH was moderately correlated with initial values of TLG $\left(r_{s}=0.4, p\right.$ value $0.01), \operatorname{MTV}\left(r_{s}=0.44, p\right.$ value 0.01$)$ and SUVmax $\left(r_{s}=0.42, p\right.$ value 0.01$)$.

Conclusion: TLG in correlation with $\mathrm{LDH}$ can be significant prognostic factors of therapeutic response in $\mathrm{HL}$. They can be used for the identification of a subset of HL patients with a better outcome.
\end{abstract}

Keywords: Hodgkin Lymphoma (HL), Positron emission tomography (PET), Lactate dehydrogenase (LDH), Correlation

\section{Background}

Lymphomas are one of the most common solid tumors [1]. The role of imaging in management of lymphoma is essential [2]. Currently, positron emission tomographycomputed tomography (PET/CT) has prominent applications as staging and during treatment to evaluate the individual chemosensitivity and subsequently to adapt further therapy by using Deauville scale [3].

Functional studies with quantitative parameters play a great role in oncologic management. The universal

\footnotetext{
*Correspondence: hend1980.hy@gmail.com

1 Department of Radiology, Om Elmasreen Hospital, Ministry of Health,

Salah Salem, Rabaa, Giza, Egypt

Full list of author information is available at the end of the article
}

metabolic quantitative parameter is the maximum standardized uptake value (SUVmax), which reflects the tumor glucose metabolism of the most aggressive cell component [4]. However, it is retrieved from only one voxel which makes it sensitive to image noise and impacted by various patient characteristics and imaging parameters. On the other hand, metabolic tumor volume (MTV) is a measurement of the viable tumor fraction and can better estimate tumor burden. The product of multiplying the mean SUV and the MTV yields the total lesion glycolysis (TLG) representing the metabolic burden of disease that depends on both tumor volume and glucose utilization rate. Thus, MTV or TLG may provide additional valuable information for prediction of tumor reaction to treatment [5]. Most studies are concerned with whole-body
Springer Open

(C) The Author(s) 2022. Open Access This article is licensed under a Creative Commons Attribution 4.0 International License, which permits use, sharing, adaptation, distribution and reproduction in any medium or format, as long as you give appropriate credit to the original author(s) and the source, provide a link to the Creative Commons licence, and indicate if changes were made. The images or other third party material in this article are included in the article's Creative Commons licence, unless indicated otherwise in a credit line to the material. If material is not included in the article's Creative Commons licence and your intended use is not permitted by statutory regulation or exceeds the permitted use, you will need to obtain permission directly from the copyright holder. To view a copy of this licence, visit http://creativecommons.org/licenses/by/4.0/. 
MTV (WBMTV) and whole-body TLG (WBTLG), with few available studies on target lesions' MTV and TLG.

In this study, we investigated the predictive value of both target lesions' MTV and TLG instead of WBMTV and WBTLG (which are time consuming and need advanced software) by using the new response evaluation criteria in lymphoma 2017 [6].

Moreover, despite the availability of multiple studies searching the correlation between TLG and MTV with Lactate Dehydrogenase (LDH) in various types of malignancies and lymphoma; yet, there were few available studies concerning Hodgkin Lymphoma (HL) [7]. We investigated the association of TLG and MTV with LDH in HL patients.

\section{Materials and methods}

After ethical committee approval, this prospective and prognostic study was applied in the period from July 2017 till December 2019 at Ain Shams University hospital. A total of 40 consecutive patients with biopsy proven $\mathrm{HL}$ underwent two PET/CT evaluations, one initial (prior to therapy) and other interim imaging (after 2-4 cycles with 3-4 months interval according to each patient's treatment protocol) using RECIL criteria as the golden standard of the current study. B-symptoms (weight loss more than $10 \%$, fever and night sweats), laboratory data (LDH and erythrocyte sedimentation rate (ESR)) and histological specimen were registered from patients' medical records.

\section{Inclusion criteria}

Any patient presented with HL who did not receive any therapy, aged 19 years or older with no sex predilection was included.

\section{Exclusion criteria}

Those who were previously treated with chemotherapy, radiotherapy patients or who underwent any surgical intervention as line of treatment, with blood glucose level more than $200 \mathrm{mg} / \mathrm{dl}$, with known allergy to intravenous contrast material or with renal impairment were excluded.

\section{Patient preparation}

Pre-procedural assessment of patient's weight and height. Blood glucose level of the both PET/CT scans in diabetic patients was adjusted by the physician to be in the range of $110-160 \mathrm{mg} / \mathrm{dl}$ at the time of examination. Additionally serum creatinine was done before the study and confirmed to be within normal range of $0.7-1.4 \mathrm{mg} / \mathrm{dL}$.

\section{${ }^{18}$ F-FDG PET/CT imaging}

Initial and interim PET/CT were performed using combined PET/CT machine (Discovery IQ 5 ring GE). The imaging protocol included physical, dietary and laboratory instructions like fasting for a minimum of $6 \mathrm{~h}$ and controlling serum glucose level [6]. Each patient was injected $0.1 \mathrm{mCi} / \mathrm{Kg}$ of $18 \mathrm{~F}$-FluorodeoxyGlucose $\left({ }^{18} \mathrm{~F}\right.$ FDG) intravenously 50-60 min before image acquisition [8].

Mid-skull to the level of the mid-thighs contrastenhanced PET/CT was performed. Low dose attenuation correction CT was used and about $90 \mathrm{ml}$ of non-ionic iodinated contrast material was injected; then, CT study was done $90 \mathrm{~s}$ after contrast administration [9]. The entire patients PET scanning was performed by the three-dimensional acquisition mode. [10]. Reconstruction of PET image data sets using CT data for attenuation correction was done and co-registered images were displayed [11].

\section{Image analysis}

Both the initial and interim CT and PET/CT scans were analyzed by 2 experienced radiologists with at least 5 -year experience in nuclear medicine field and decision was reached by consensus. The images were evaluated for whole-body detection, follow-up of FDG-avid lymph nodes and extra-nodal lesions as well as detection of any new FDG-avid lesions.

The assessment of tumor burden in lymphoma was performed using contrast-enhanced CT images by the sum of longest diameters of a maximum of three target lesions where the three largest ones being selected and preferably representing multiple sites or organs (Fig. 1) [6].

All PET/CT fused images were analyzed for visual assessment and quantitative FDG uptake measurements (Fig. 1). The SUVmax was determined as the highest SUV of the pixel in the region of interest (ROI). Either the liver background SUVmax or mediastinal blood pool SUVmax was calculated for each patient as an internal references with comparison of the tumor activity and avidity. Initial MTV, summing the volumes of target lesions, was computed using the SUVmax threshold of $41 \%$. The TLG was calculated using the following equation: $\mathrm{TLG}=\mathrm{MTV} \times \mathrm{SUVmean}$. All parameters were corrected for Lean Body Mass (LBM) (Fig. 2) [12].

According to the RECIL classification, the interpretation of scans of lesions with FDG-avid profiles was by Deauville 5-point scale [6].

Regarding the splenic involvement, focal lesions was diagnosed by increased FDG uptake more than the splenic background [13]. Also hepatic involvement was 


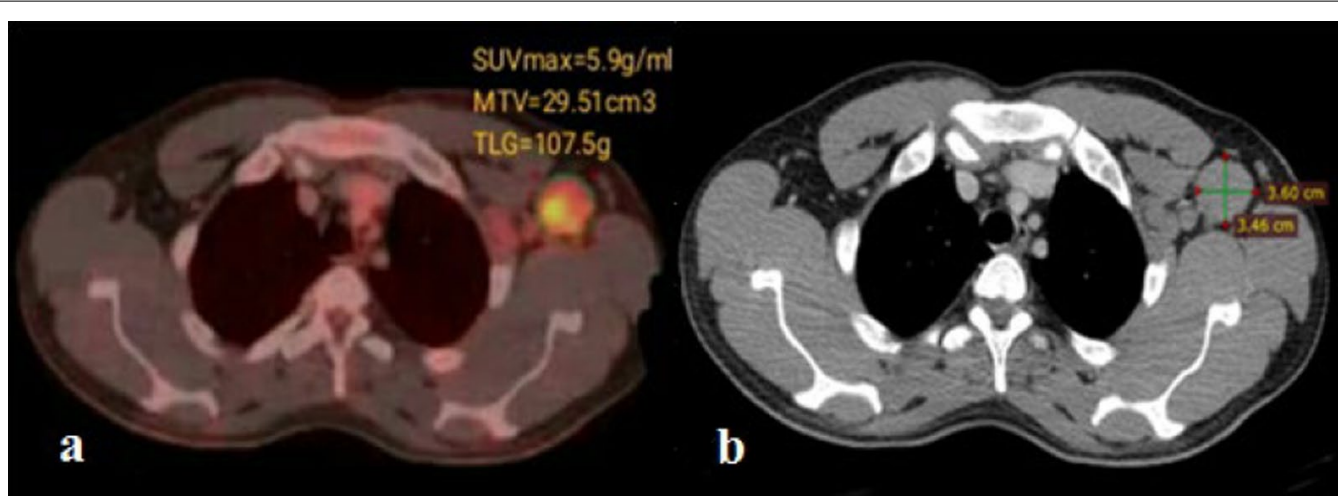

Fig. 1 A male patient, 31 years old, presented with $H L$ a Axial CE CT image shows the dimensions of left axillary LN $3.6 \times 3.46 \mathrm{~cm}$. b Axial fused image of PET/CT shows the quantitative assessment of left axillary LN SUVmax $=5.9 \mathrm{~g} / \mathrm{ml}, \mathrm{MTV}=29.51 \mathrm{~cm}^{3}$ and TLG $=107.5 \mathrm{~g}$

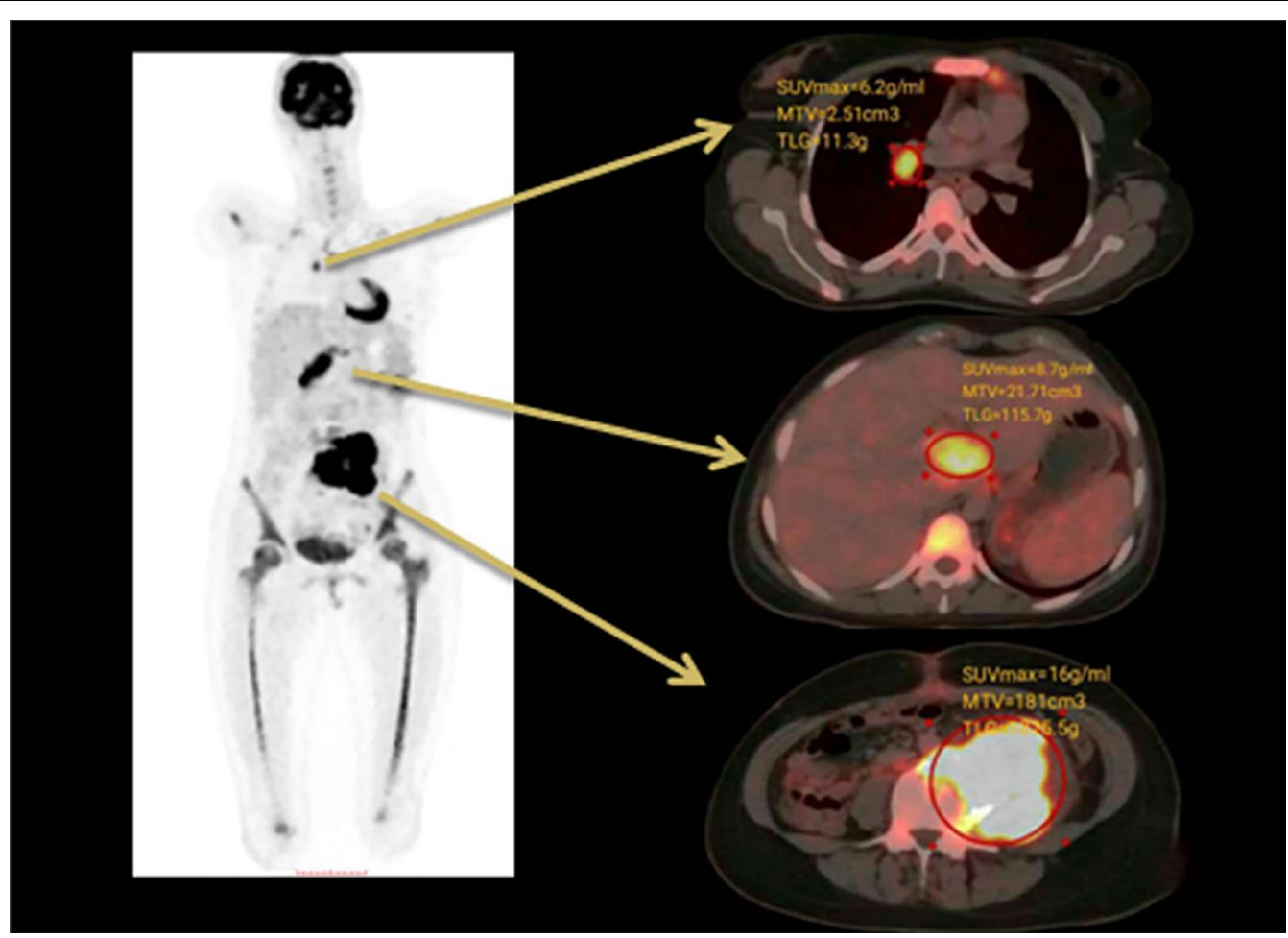

Fig. 2 Coronal MIP image and Axial fused images of PET/CT of a female patient, 19 years old, presented with HL showing the measurement of PET/ CT parameters of three largest target lesions at different body sites (right hilar LN with SUVmax $=6.2 \mathrm{~g} / \mathrm{ml}, \mathrm{MTV}=2.51 \mathrm{~cm}^{3}$ and TLG $=11.3 \mathrm{~g}, \mathrm{porta}$ hepatis $L N$ with SUVmax $=8.7 \mathrm{~g} / \mathrm{ml}, M T V=21.71 \mathrm{~cm}^{3}$ and TLG $=115.7 \mathrm{~g}$ and left paravertebral retroperitoneal soft tissue lesion with SUVmax=16 g/ $\mathrm{ml}, \mathrm{MTV}=181 \mathrm{~cm}^{3}$ and $\mathrm{TLG}=1825.5 \mathrm{~g}$ )

diagnosed by increased focal concentration of FDG against a background of relatively lower normal hepatocyte uptake [14]. Bone marrow involvement was either diffusely increased which is mostly nonspecific, or focally increased that denotes marrow lesions [15].

Initial and interim studies were compared to determine the radiological response after receiving chemotherapy.
According to the RECIL 2017 in response assessment, the patients were classified to two groups $\mathrm{CR}$ and $\mathrm{nCR}$ groups. The nCR was an umbrella term encompassing all response patterns other than $\mathrm{CR}$, as described by the RECIL criteria while CR was a term applied on all patients who showed complete disappearance of either all target lesions and all lymph nodes with a long axis less 
than $10 \mathrm{~mm}$ or $30 \%$ or more decrease in sum of longest diameters of target lesions with normalization of FDG PET (Deauville score 1-3) with neither bone marrow involvement nor new lesions. On the other hand, $\mathrm{nCR}$ is a term applied on all patients who showed either $30 \%$ or more decrease in sum of longest diameters of target lesions with positive FDG PET (Deauville score 4-5), or less than $30 \%$ reduction, or any increase in the summation of longest dimensions of the target lesions with any FDG PET findings associated with bone marrow involvement or new lesion [16].

As a part of the staging procedures in HL patients, serum LDH was routinely determined by the in-house laboratory. The tumor marker LDH was extracted from the clinical data base within 7 days before, up to 7 days after PET/CT. The upper limit of the reference range is $240 \mathrm{U} / \mathrm{L}$.

In addition, we explored the possibility of association between the serum LDH and target lesions' TLG and MTV by direct correlation of their absolute values.

\section{Statistical analysis}

Statistical analysis was performed using MedCalc statistical software for Windows (MedCalc Software, Mariakerke, Belgium). Data for initial SUV, TLG and MTV were expressed as median and range while data for initial LDH, ESR and B-symptoms were expressed as both number and percentage. Mann-Whitney $U$ test was used to evaluate the differences of initial TLG, MTV and SUV between $\mathrm{CR}$ and $\mathrm{nCR}$ groups. Receiver operator characteristic (ROC) curve analysis was performed to determine optimum thresholds and the diagnostic accuracy of the initial SUV and TLG in distinguishing the different groups. The diagnostic accuracy of both initial SUV and TLG was evaluated in terms of sensitivity, specificity, positive predictive value (PPV), negative predictive value (NPV), and area under the ROC curve (AUC). Spearman's rank correlation was used to describe the correlations between the initial LDH and the initial PET/CT parameters (SUV, TLG and MTV). Chi-squared test was used for comparison of initial LDH, ESR and B-symptoms between the two groups. For all tests, all $\mathrm{P}$ values were two-tailed and a $p$ value $<0.05$ was considered significant.

\section{Results}

\section{Clinical and demographic data of the studied patients}

Initial demographic, clinical and pathologic characteristics of 40 patients are listed in Table 1. (21 males and 19 females). The median age was 30.5 years old ages (range, 19-85 years old). A maximum of three largest target lesions was assessed per patient with a total of 110 lesions in 40 patients. Twenty-six patients (65\%) showed
Table 1 Clinical and demographic data of the studied patients

\begin{tabular}{|c|c|}
\hline \multicolumn{2}{|l|}{$n=40$} \\
\hline Age, median (range) & $\begin{array}{l}30.5 \text { years } \\
(19- \\
85 \text { years })\end{array}$ \\
\hline \multicolumn{2}{|l|}{ Gender } \\
\hline Male & 21 \\
\hline Female & 19 \\
\hline \multicolumn{2}{|l|}{ B symptoms } \\
\hline Positive & 24 \\
\hline Negative & 16 \\
\hline \multicolumn{2}{|l|}{$\mathrm{LDH}$} \\
\hline Normal & 4 \\
\hline Elevated & 36 \\
\hline \multicolumn{2}{|l|}{ ESR } \\
\hline Normal & 0 \\
\hline Elevated & 40 \\
\hline \multicolumn{2}{|l|}{ Ann Arbor stage } \\
\hline I & 8 \\
\hline$\|$ & 18 \\
\hline III & 6 \\
\hline IV & 8 \\
\hline \multicolumn{2}{|l|}{ Histology } \\
\hline Nodular sclerosis & 24 \\
\hline Mixed cellularity & 16 \\
\hline \multicolumn{2}{|l|}{ Target lesions } \\
\hline LNs & 75 \\
\hline Other lesions & 17 \\
\hline Splenic lesions & 10 \\
\hline Bone lesions & 8 \\
\hline \multicolumn{2}{|l|}{ RECIL response to $\mathrm{CTH}$} \\
\hline Complete response & 26 \\
\hline Partial response* & 8 \\
\hline Minor response $e^{* *}$ & 4 \\
\hline Stable disease & 0 \\
\hline Progressive disease & 2 \\
\hline
\end{tabular}

*Partial Response means 30\% or more reduction of the summation of longest dimensions of the target lesions but not a complete response with Positive FDG-PET findings

**Minor Response means between 10 and $30 \%$ reduction of the summation of longest dimensions of the target lesions with FDG-PET findings

CR while fourteen (35\%) showed a nCR on interim imaging (Figs. 3, 4, 5).

\section{PET parameters Predictive ability}

The initial PET metabolic parameters including SUVmax, MTV and TLG of both CR and nCR groups are listed in Table 2. Patients with CR were found to significantly had lower levels of SUVmax and TLG compared to patients with $\mathrm{nCR}$ ( $p$ value $0.03,0.047$, respectively). 


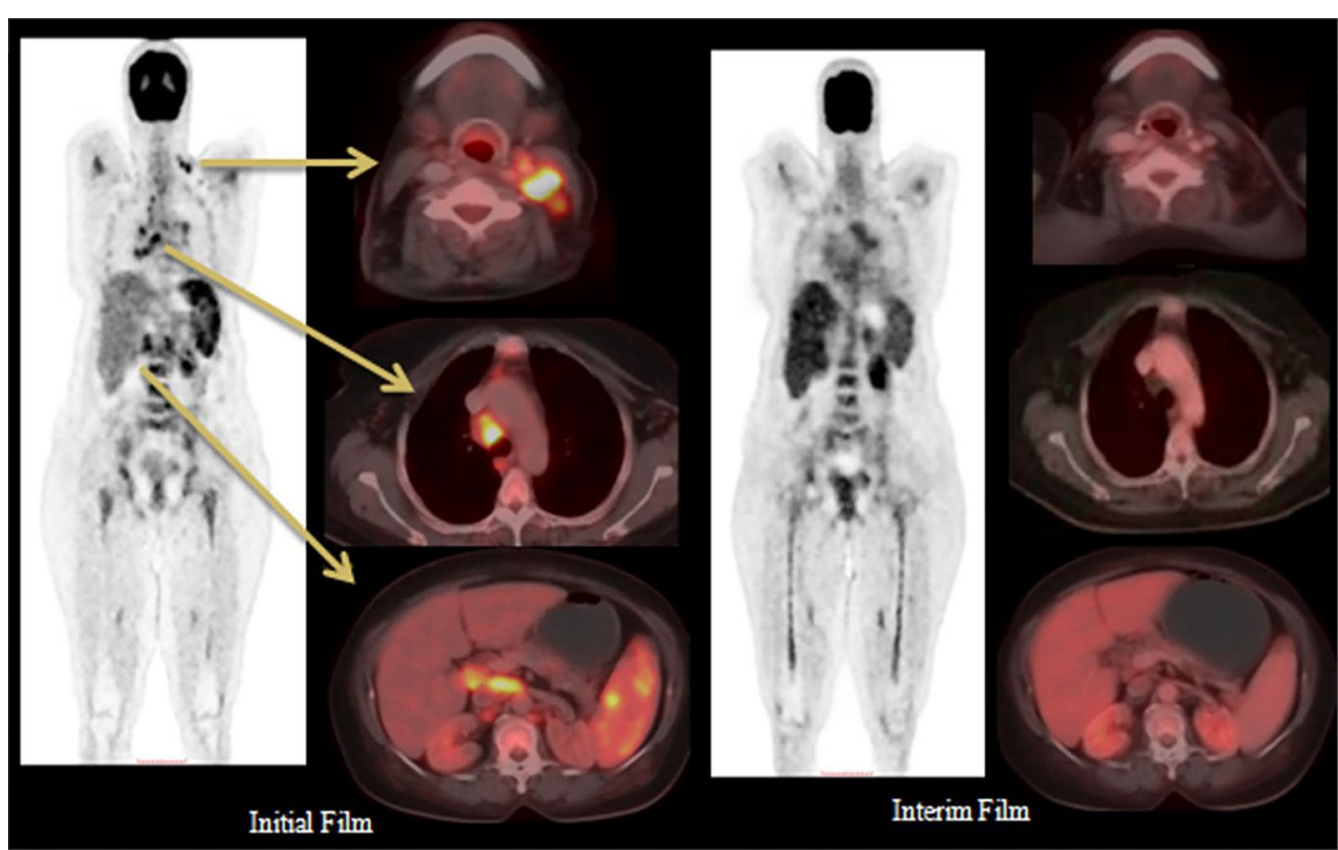

Fig. 3 Coronal MIP images and Axial fused images of PET/CT of a female patient, 47 years old, presented with stage III HL. On the left, the initial film shows three target lesions (left cervical level IV LN with $S U V \max =6.38 \mathrm{~g} / \mathrm{ml}, M T V=2.64 \mathrm{~cm}^{3}$ and TLG $=9.9 \mathrm{~g}$, amalgamated retrocaval paratracheal $L N$ with SUVmax $=6.96 \mathrm{~g} / \mathrm{ml}, \mathrm{MTV}=9.97 \mathrm{~cm}^{3}$ and $T L G=38.9 \mathrm{~g}$ and preaortic and precaval $L N s$ with $S U V \max =6.49 \mathrm{~g} / \mathrm{ml}, \mathrm{MTV}=24.57 \mathrm{~cm}{ }^{3}$ and $T L G=90.6 \mathrm{~g}$. On the right, the interim film shows complete anatomical and metabolic clearance of the lesions on the left side (CR group)

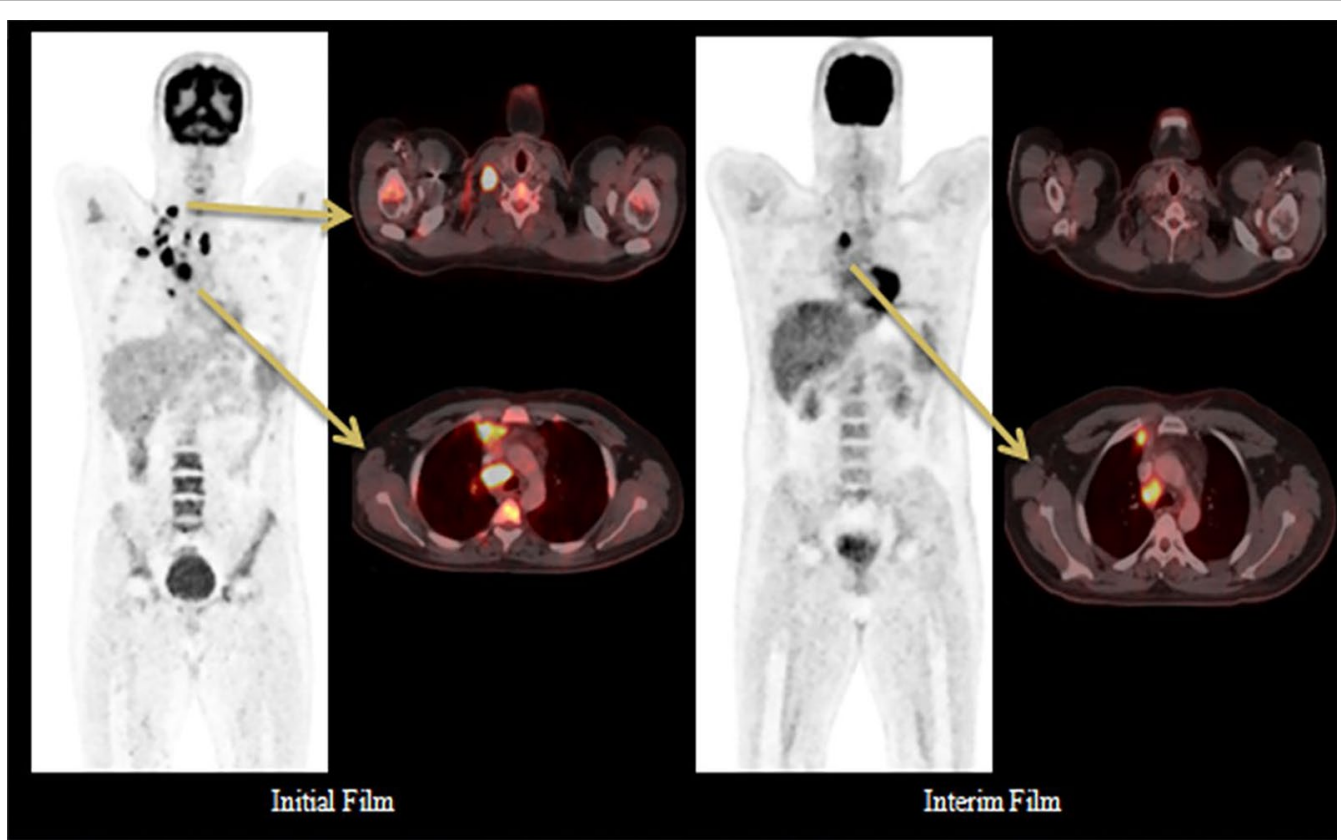

Fig. 4 Coronal MIP images and Axial fused images of PET/CT of a male patient, 24 years old, presented with stage II HL. On the left, the initial film shows two target lesions (right Supraclavicular LN with SUV max $=21.35 \mathrm{~g} / \mathrm{ml}, \mathrm{MTV}=4.59 \mathrm{~cm}^{3}$ and TLG $=61 \mathrm{~g}$ and pretracheal retrocaval LNs with SUV max $=23.1 \mathrm{~g} / \mathrm{ml}, \mathrm{MTV}=16.16 \mathrm{~cm}^{3}$ and $T L G=227 \mathrm{~g}$ ). On the right, the interim film shows complete metabolic resolution with marked anatomical regression of right Supraclavicular $L N$ while the other shows SUV $\max =7.4 \mathrm{~g} / \mathrm{ml}, \mathrm{MTV}=8.02 \mathrm{~cm}^{3}$ and TLG $=37 \mathrm{~g}$ denoting partial metabolic response (non-CR group) 


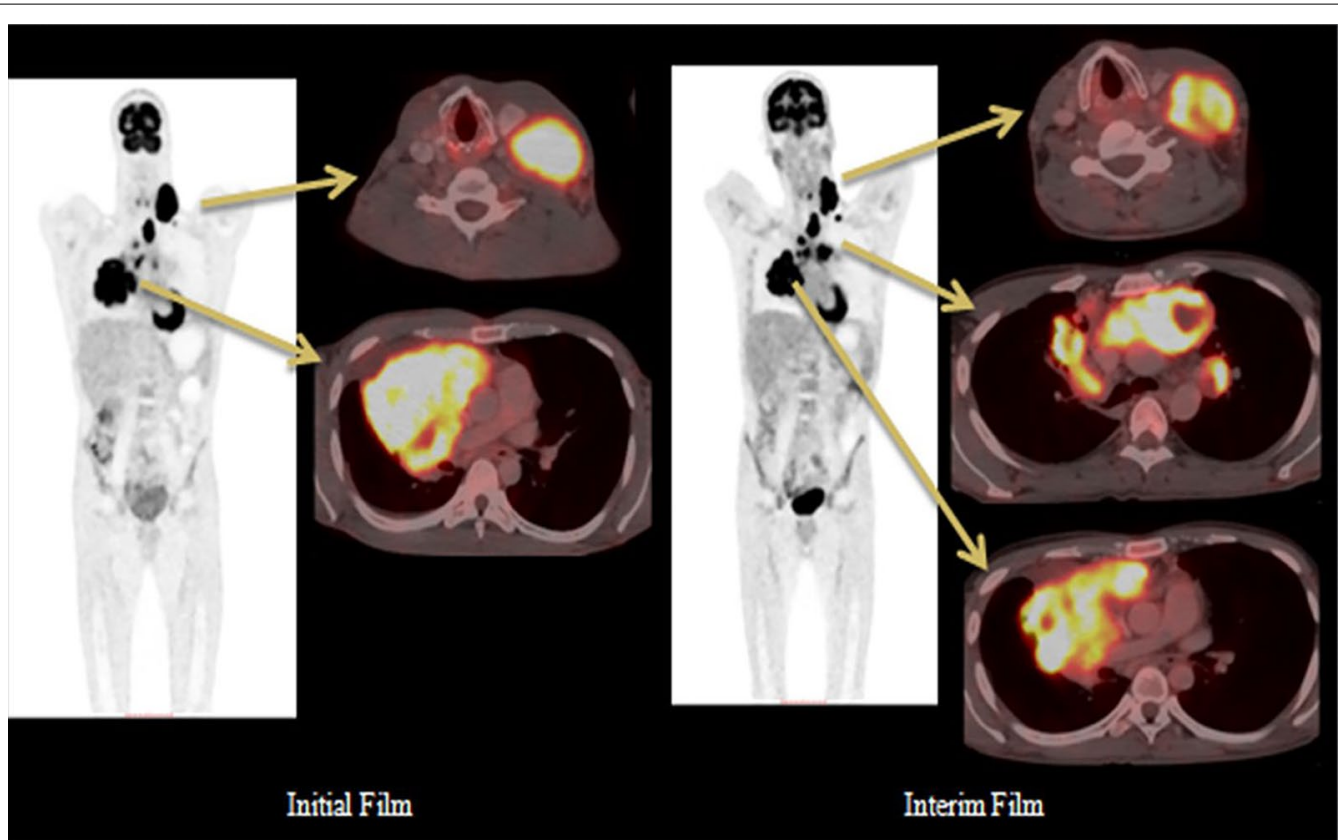

Fig. 5 Coronal MIP and Axial fused images of PET/CT of a male patient, 32 years old, presented with stage II HL. On the left, the initial film shows two target lesions (left cervical amalgamated LN with SUV $\max =11.2 \mathrm{~g} / \mathrm{ml}, \mathrm{MTV}=28.7 \mathrm{~cm}^{3}$ and TLG $=279.7 \mathrm{~g}$ and amalgamated mediastinal nodal mass with SUV $\max =12.2 \mathrm{~g} / \mathrm{ml}, \mathrm{MTV}=3624 \mathrm{~cm}^{3}$ and TLG $=2777.9 \mathrm{~g}$ ). On the right, the interim film shows SUV $\max =12 \mathrm{~g} / \mathrm{ml}, \mathrm{MTV}=69.16 \mathrm{~cm}^{3}$ and TLG $=552.2 \mathrm{~g}$ of cervical lesion, SUV $\max =11.3 \mathrm{~g} / \mathrm{ml}, \mathrm{MTV}=165 \mathrm{~cm}^{3}$ and $T L G=1605.7 \mathrm{~g}$ of mediastinal lesion and denovo left hilar LN SUV $\max =11 \mathrm{~g} / \mathrm{ml}, \mathrm{MTV}=2.95 \mathrm{~cm}^{3}$ and $\mathrm{TLG}=28.9 \mathrm{~g}$ denoting progressive metabolic response (non-CR group)

Table 2 Comparison between quantitative PET/CT parameters of the $C R$ and $n C R$ groups

\begin{tabular}{|c|c|c|c|}
\hline & CR & $\mathrm{nCR}$ & $p$ value \\
\hline \multicolumn{4}{|c|}{ Initial SUVmax } \\
\hline Median & 18.3 & 30.3 & $p=0.03^{*}$ \\
\hline Range & $4.7-74.4$ & $13.6-108.3$ & \\
\hline \multicolumn{4}{|l|}{ Initial TLG } \\
\hline Median & 138.0 & 288 & \\
\hline Range & $26.1-1613.4$ & $57.9-3057.6$ & $p=0.047^{*}$ \\
\hline \multicolumn{4}{|l|}{ Initial MTV } \\
\hline Median & 29.5 & 30.8 & \\
\hline Range & $2.7-203.3$ & $8.8-390.5$ & $p=0.26$ \\
\hline
\end{tabular}

*Statistically significant
No significant association was found between the initial MTV of both groups ( $p$ value 0.26 ) (Table 2).

Their optimum threshold values to differentiate between CR and nCR groups were 19.52 for SUVmax and 158.6 for TLG with similar sensitivity, specificity, positive predictive value (PPV), negative predictive value (NPV), accuracy and area under the curve (AUC) (Table 3).

Regarding their diagnostic performance in distinguishing CR group from nCR group, there was no statistically significant difference between initial SUV and initial TLG ( $p$ value 0.82 ) (Fig. 6).

\section{LDH correlation to PET parameters}

The initial LDH was moderately associated with initial values of TLG $\left(r_{\mathrm{s}}=0.4, p\right.$ value 0.01$)$, MTV $\left(r_{\mathrm{s}}=0.44, p\right.$ value 0.01$)$ and SUVmax $\left(r_{\mathrm{s}}=0.42, p\right.$ value 0.01$)$.

Table 3 The diagnostic accuracy of quantitative PET/CT parameters for differentiation of CR from the nCR group

\begin{tabular}{lllllllll}
\hline Parameter & $\begin{array}{l}\text { Optimum } \\
\text { threshold value }\end{array}$ & Sensitivity (\%) & Specificity (\%) & PPV (\%) & NPV (\%) & Accuracy (\%) & AUC & $p$ value \\
\hline Initial SUVmax & $\leq 19.52$ & 53.9 & 85.7 & 87.5 & 50 & 65 & 0.71 & $0.01^{*}$ \\
Initial TLG & $\leq 158.6$ & 53.9 & 85.7 & 87.5 & 50 & 65 & 0.69 & $0.036^{*}$ \\
\hline
\end{tabular}

\footnotetext{
${ }^{*}$ Statistically significant
} 


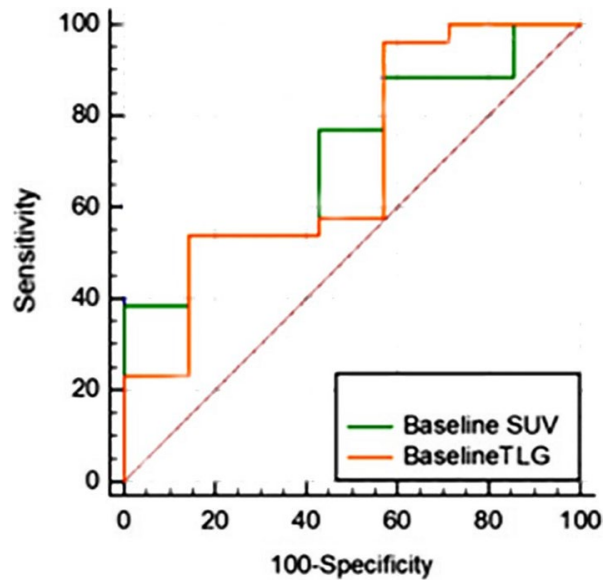

Fig. 6 Receiver operating characteristic curve analyses. The specificity and sensitivity of using baseline SUV and baseline TLG in differentiating $C R$ and non-CR groups were compared. No significant differences existed between the AUCs of baseline SUV and baseline TLG

\section{Clinical characteristics predictive ability}

Other factors including initial LDH, ESR showed no significant association between their levels and treatment response $(\times 2=2.33,3.6$ and $p$ value $0.13,0.06$, respectively) whereas B-symptoms were associated with treatment response where patients with no B symptoms at initial evaluation were significantly more prevalent in the CR group as opposed to the nCR group $(\times 2=5.79$, $p$ value 0.02 ).

\section{Discussion}

To improve HL treatments, the recent studies goal is to achieve the best chance of curing well with the least possible long-term risks of chemotherapy. Subsequently, modern approaches have attempted to investigate PET/ CT parameters predictive ability to define subgroups of patients who will show early response after start of therapy [17]. The most commonly studied parameter is SUVmax, but unfortunately, it reflects the metabolic activity of the most aggressive tumor cell [5], while the functional imaging parameters (MTV and TLG) overcome SUVmax limitation as they evaluate the total volume and metabolic activity of tumor cells [18]. In our study, the predictive value of the initial quantitative indices of $\mathrm{PET} / \mathrm{CT}$ (SUVmax, TLG, MTV) has been evaluated during the management of HL.

As many criteria were developed to assess the tumors therapeutic response, Tawfik et al. reported that PET response criteria in solid tumor (PERCIST) had improved the PET/CT predictive value [19]. So we adopt a new RECIL criteria with a simple approach of estimating PET/CT parameters' values from maximum three largest target lesions rather than the whole-body approach. We have found that the initial SUVmax and TLG showed a good predictive value to distinguish between the patients with CR from those with nCR.

Similarly, the available literature has demonstrated the predictive value of TLG as an effective tool for response evaluation in FDG-avid lymphoma. Wang et al. reported that initial TLG tends to be superior to SUVmax and MTV in predicting survival [20]. Moustafa et al. suggested that TLG is an independent predictor of survival in malignant lymphoma especially in high risk patients and may be an influential predictor of lymphoma outcome [21].

Pike et al. and Zaucha et al. considered TLG as a very strong prognostic parameter which can improve the predictive value of $\mathrm{PET} / \mathrm{CT}$ and was associated with interim PET response [22, 23]. El-Galaly et al. and Guo et al. suggested that high initial total TLG predicts significantly worse progression $[24,25]$.

Our threshold value of TLG is much lower than that of previous work by Pike et al. and Akhtari et al. who reported WBTLG cutoff value of 3318 in advanced HL and 1703 in early-stage HL, respectively. It is clear that this is variation is due to different methods of tumor burden assessment. However, our cutoff value requires more validation $[22,26]$.

Both initial SUVmax and TLG have similar sensitivity, specificity, accuracy, ROC as a diagnostic tool in distinguishing the $\mathrm{CR}$ from the $\mathrm{nCR}$. We deduce that patients with low initial target lesions' TLG will have a better outcome than those with higher values. We suggest that TLG can be used as an alternative to SUVmax because SUVmax provides limited semiquantitative estimate of single voxel activity of a tumor mass and is affected by various factors like image noise, uptake period, body composition and plasma glucose level [5].

The study by Pike et al. was inconsistent with our results, where they demonstrated the predictive value of MTV as an effective tool for response evaluation. They measured the initial total MTV and MTV of the bulkiest lesion in advanced HL patients to evaluate whether both could predict prognosis and interim PET response. They reported that initial MTV is associated with interim PET response [22].

Moustafa et al. suggested that MTV is an independent predictor of malignant lymphoma survival and outcome [21].

Also El-Galaly et al., Guo et al. and Kanoun et al. studies were different with our results as they reported that tumor burden quantification by initial WBMTV may enhance prognostication and proved that lymphoma 
patients with a low initial MTV had a better prognosis than those with a high MTV [24, 25, 27].

Zaucha et al. and Cottereau et al. considered MTV as a very strong prognostic parameter being superior to the available parameters [23, 28].

On the contrary, our results show that the initial MTV does not have significant difference between patients with CR and nCR. This difference may be attributed to the methodological differences regarding tumor burden assessment. We adopted quantitative assessment of only maximum three largest target lesions, while other investigators measured total MTV.

Unlike the three target lesion approach, the wholebody approach may more reliably reflect the patient's tumor status and treatment response in terms of MTV assessment. Son et al. and Pellegrino et al. stated that using the sum of PET/CT parameter of all patient's lesions was more expressive of the metabolically active tumor volume than measuring it at one site $[29,30]$.

Elevated LDH is associated with high tumor burden as cancer cells are dependent on anaerobic metabolism with subsequent production of lactate from glucose (Warburg effect) [31].

Subsequently, LDH can be used to monitor response to chemotherapy for certain types of cancers like lymphoma by assessing it at diagnosis, after treatment, and during follow-up [31]. Although there are few studies correlating LDH to SUVmax [7], to the best of our knowledge, there are no reports investigating the correlation of LDH with TLG or MTV in HL.

We have found a significant correlation between the initial LDH and initial SUVmax, MTV and TLG. A possible explanation for this correlation is attributed to the major role of nicotinamide adenine dinucleotide reduced/ nicotinamide adenine dinucleotide oxidized (NADH/NAD+) homeostasis in various metabolic reactions including glucose metabolism whereas NADH/NAD+ are up regulated in tumor cells in order to maintain its survival reduction of pyruvate to lactate (the Warburg effect) by LDH to produce $\mathrm{NAD}+[32]$.

This increased glucose metabolism accounts for FDG accumulation into cancer cells and subsequently all quantitative parameters especially TLG which is theoretically superior to other measurements because it incorporates both parameters by taking into account the level of glucose accumulation within the total volume of all the regions of all ROIs [33].

Our finding regarding SUVmax correlation with LDH is consistent with Ucar et al. who found in 34 patients -13 patients had HL- that end of treatment (EOT) SUVmax are related to the EOT laboratory parameters such as LDH [34] and inconsistent with Li et al. who found in 103 patients -19 had HL- that SUVmax at biopsy site was not significantly correlated with LDH [7].

This study was limited by few limitations. First, its short duration including only interim PET/CT studies with unavailability of EOT. Functional parameters of EOT would have given more comprehensive evaluation of the quantitative indices. However, the retrieved quantitative indices of initial scans could give insights into prognosis and the effectiveness of therapy. Second, the heterogeneous patient population in terms of the pathological subtypes and chemotherapy regimen. More prospective studies dedicated to specific pathological subtypes and treatment regimen will be more appropriate for proper functional indices assessment.

\section{Conclusion}

In conclusion, the initial three target lesions' TLGbased on RECIL criteria-in correlation with LDH can predict early interim therapeutic response in HL patients and may be used for identification of a subset of patients with a better outcome which subsequently may help later in decision making and management adjustment of these patients after more future studies to be with highest curative chance and the least possible long-term risks.

\section{Abbreviations \\ SUVmax: Maximum standardized uptake value; LDH: Lactate dehydrogenase; HL: Hodgkin's lymphoma; TLG: Total lesion glycolysis; MTV: Metabolic tumor volume; PET: Positron emission tomography; NHL: Non-Hodgkin's lymphoma; 18F-FDG: 18F-FluorodeoxyGlucose; CT: Computed tomography; WBMTV: Whole-body metabolic tumor volume; WBTLG: Whole-body total lesion glycolysis; RECIL: Response evaluation criteria in lymphoma; ESR: Erythrocyte sedimentation rate; mg: Milligram; dl: Deciliter; mCi: Microcuries; Kg: Kilogram; LBM: Lean body mass; CR: Complete response; nCR: Non-complete response; ROI: Region of interest; AUC: Area under the curve; ROC: Receiver operating characteristic; PPV: Positive predictive value; NPV: Negative predictive value; $R_{s}$ : Spearman rank correlation coefficient; LNs: Lymph nodes; CTH: Chemo- therapy; PERCIST: PET response criteria in solid tumor; NADH: Nicotinamide adenine dinucleotide reduced; NAD+: Nicotinamide adenine dinucleotide oxidized; EOT: End of treatment.}

\section{Acknowledgements}

Not applicable.

\section{Authors' contributions}

HY participated in data collection, image interpretations, statistical analysis and writing manuscript. SA participated in the design of study data collection, statistical analysis, editing, and supervision. AH participated in the design of study, editing, and supervision. AM participated in the design of study, editing, and supervision. $\mathrm{MH}$ participated in the data collection, image interpretation, editing, and supervision. All authors read and approved the final manuscript.

\section{Funding}

No funding to declare.

\section{Availability of data and materials}

The datasets generated and analyzed during the current study are not publicly available due to individual privacy but are available from the corresponding author on reasonable request. 


\section{Declarations}

\section{Ethics approval and consent to participate}

The study was approved by the ethical committee of "Research Ethics Committee at the Faculty of Medicine, Ain Shams University" with ethical committee approval number FMASU M D 235/2018. An informed written consent was taken from all subjects.

\section{Consent for publication}

The institutional review board of our institute approved this study, and the requirement to obtain informed consent was waived.

\section{Competing interests}

The authors declare that they have no competing interests.

\section{Author details}

1 Department of Radiology, Om Elmasreen Hospital, Ministry of Health, Salah Salem, Rabaa, Giza, Egypt. ${ }^{2}$ Department of Radiology, Faculty of Medicine, Ain Shams University Hospitals, Ramsis street, Abbasia, Cairo, Egypt.

\section{Received: 23 August 2021 Accepted: 10 January 2022}

Published online: 21 January 2022

\section{References}

1. Singh R, Shaik S, Negi BS, Rajguru JP, Patil PB, Parihar AS et al (2020) NonHodgkin's lymphoma: a review. J Family Med Prim Care 9:1834-1840

2. Rutherford SC (2019) Surveillance Scanning in Lymphoma. Clin Adv Hematol Oncol 17:352-359

3. Voltin CA, Mettler J, Grosse J, Dietlein M, Baues C, Schmitz C et al (2020) FDG PET imaging for Hodgkin and diffuse large B-cell lymphoma-an updated overview. Cancers 12:601-615

4. Im HJ, Bradshaw T, Cho SY (2018) Current methods to define metabolic tumor volume in positron emission tomography: which one is better? Nucl Med Mol Imaging 52:5-15

5. Xie M, Zhai W, Cheng S, Zhang H, Xie Y, He W (2016) Predictive value of F-18 FDG PET/CT quantization parameters for progression free survival in patients with diffuse large B-cell lymphoma. Hematology 21:99-105

6. Younes A, Hilden P, Coiffier B, Hagenbeek A, Salles G, Wilson W et al (2017) International Working Group consensus response evaluation criteria in lymphoma (RECIL 2017). Ann Oncol 28:1436-1447

7. Li H, Wang X, Zhang L, Yi X, Qiao Y, Jin Q (2019) Correlations between maximum standardized uptake value measured via ${ }^{18} \mathrm{~F}$-fluorodeoxyglucose positron emission tomography/computed tomography and clinical variables and biochemical indicators in adult lymphoma. J Cancer Res Ther 15:1581-1588

8. Ulaner GA (2019) Fundamentals of oncologic PET/CT. United States, Philadelphia

9. Swanston NM, Christian PE (2012) Clinical PET/CT in oncology. In: Christian PE, Waterstram-Rich KM (ed) Nuclear medicine and PET/CT technology and techniques, 7th edn. St. Louis, United States

10. Basu S, Kwee KC, Surti S, Akin EA, Yoo D, Alavi A (2011) Fundamentals of PET and PET/CT imaging. Ann NY Acad Sci 1228:1-18

11. Abd El-Aziz EM, Abd Elfattah A, Sayed HM (2019) The clinical utility of PET/CT in breast cancer management and follow up. Acta Sci Med Sci 7:23-39

12. Albano D, Bosio G, Tomasini D, Bonù $M$, Giubbini $R$, Bertagna $F$ (2020) Metabolic behavior and prognostic role of pretreatment ${ }^{18} \mathrm{~F}$ FDG PET/CT in gist. Asia-Pac J Clin Oncol 16:207-221

13. Berzaczy D, Haug AR, Raderer M, Kiesewetter B, Berzaczy G, Weber M et al (2019) Is there a reliable size cut-off for splenic involvement in lymphoma? A ${ }^{18}$ F FDG PET controlled study. PLOS ONE. https://doi.org/ 10.1371/e0213551

14. Rachh S, Basu S (2014) PET/CT in patients with liver lesions of different nature. Clin Transl Imaging 2:139-155

15. Perry C, Lerman H, Joffe E, Sarid N, Amit O, Avivi I et al (2016) The value of $\mathrm{PET} / \mathrm{CT}$ in detecting bone marrow involvement in patients with follicular lymphoma. Medicine 95:1-7
16. Boellaard R, Delgado-Bolton R, Oyen WJG, Giammarile F, Tatsch K, Eschner W et al (2015) FDG PET/CT: EANM procedure guidelines for tumor imaging version 2.0. Eur J Nucl Med Mol Imaging 42:328-354

17. Shanbhag S, Ambinder R (2018) Hodgkin Lymphoma: a review and update on recent progress. CA Cancer J Clin 68:116-132

18. Matsumoto Y, Baba S, Endo M, Setsu N, lida K, Fukushi J et al (2017) Metabolic tumor volume by ${ }^{18} \mathrm{~F}-\mathrm{FDG}$ PET/CT can predict the clinical outcome of primary malignant spine/spinal tumors. BioMed Res Int. https://doi. org/10.1155/2017/8132676

19. Tawfik MMH, Monib AM, Yassin A, Ali SA (2020) Comparison between RECIST and PERCIST criteria in therapeutic response assessment in cases of lymphoma. Egypt J Radiol Nucl Med 51:1-10

20. Wang H, Shen G, Jiang C, Li L, Cui F, Tian R (2018) Prognostic value of baseline, interim and end-of-treatment 18F-FDG PET/CT parameters in extranodal natural killer/T-cell lymphoma: a meta-analysis. PLOS ONE. https://doi.org/10.1371/e0194435

21. Moustafa H, Mattar M (2020) Prognostic value of volume based parameters of PET/CT in Malignant Lymphoma. Egypt J Nucl Med 21:1-7

22. Pike L, Kirkwood AA, Patrick P, Radford J, BurtonStevens C et al (2017) Can baseline PET/CT features predict outcomes in advanced Hodgkin Lymphoma? A prospective evaluation of UK patients in the RATHL trial (CRUK/07/033). Hematol Oncol 35:37-38

23. Zaucha JM, Chauvie S, Zaucha R, Biggii A, Gallamini A (2019) The role of $\mathrm{PET} / \mathrm{CT}$ in the modern treatment of Hodgkin lymphoma. Cancer Treat Rev 77:44-56

24. El-Galaly TC, Villa D, Gormsen LC, Baech J, Lo A, Cheah CY (2018) FDG-PET/ $\mathrm{CT}$ in the management of lymphomas: current status and future directions. J Intern Med 284:358-376

25. Guo B, Tan X, Ke Q, Cen H (2019) Prognostic value of baseline metabolic tumor volume and total lesion glycolysis in patients with lymphoma: a meta-analysis. PLoS ONE. https://doi.org/10.1371/e0210224

26. Akhtari M, Milgrom SA, Pinnix CC, Reddy JP, Dong W, Smith GL et al (2018) Reclassifying patients with early-stage Hodgkin lymphoma based on functional radiographic markers at presentation. Blood 131:84-94

27. Kanoun S, Rossi C, Casasnovas O (2018) ${ }^{18} \mathrm{~F} \mathrm{FDG} \mathrm{PET/CT} \mathrm{in} \mathrm{Hodgkin}$ Lymphoma: current usefulness and perspectives. Cancers 145:1-11

28. Cottereau AS, Versari A, Loft A, Casasnovas O, Bellei M, Ricci R et al (2018) Prognostic value of baseline metabolic tumor volume in early stage Hodgkin's lymphoma in the standard arm of $\mathrm{H} 10$ trial. Blood 131:1456-1463

29. Son SH, Lee SW, Jeong SY, Song B, Chae YS, Ahn BC et al (2015) Wholebody metabolic tumor volume, as determined by ${ }^{18} \mathrm{~F} \mathrm{FDG} \mathrm{PET/CT,} \mathrm{as} \mathrm{a}$ prognostic factor of outcome for patients with breast cancer who have distant metastasis. AJR Am J Roentgenol 205:878-885

30. Pellegrino S, Fonti R, Pulcrano A, Vecchio SD (2021) PET based volumetric biomarkers for risk stratification of non-small cell lung cancer patients. Diagnostics 11:1-15

31. Yadav C, Ahmad A, D'Souza B, Agarwal A, Nandini M, Prabhu Ket al (2016) Serum lactate dehydrogenase in non-Hodgkin's Lymphoma: a prognostic indicator. Ind J Clin Biochem 31:240-242

32. Thapa M, Dallmann G (2020) Role of coenzymes in cancer metabolism. Semin Cell Dev Biol 98:44-53

33. MCDonald JE, Kessler MM Gardner MW Buros AF, Ntambi JA, Waheed S et al (2017) Assessment of total lesion glycolysis by ${ }^{18} \mathrm{~F} \mathrm{FDG} \mathrm{PET/CT}$ significantly improves prognostic value of GEP and ISS in Myeloma. Clin Cancer Res 23:1981-1987

34. Ucar E, Yalcin H, Kavvasoglu GH, Ilhan G (2018) Correlations between the maximum standard uptake value of positron emission tomography/ computed tomography and laboratory parameters before and after treatment in patients with lymphoma. Chin Med J 131:1776-1779

\section{Publisher's Note}

Springer Nature remains neutral with regard to jurisdictional claims in published maps and institutional affiliations. 